Pacific Journal of Mathematic 


\title{
ULTRAFILTERS AND THE BASIS PROPERTY
}

\author{
Richard A. SANERIB, JR.
}

\begin{abstract}
Three notions of a basis for an ultrafilter in a Boolean algebra are investigated in this paper, namely having an independent set of generators, a weakly independent set of generators and a weakly independent set of generators over a proper subfilter. In general these three notions are distinct, but for a Boolean algebra with an ordered base the latter two are equivalent. This paper shows that a large class of Boolean algebras do not possess ultrafilters with a basis, in particular no infinite homomorphic image of a $\sigma$-complete Boolean algebra has a nonprincipal ultrafilter with a basis. For Boolean algebras with an ordered base necessary and sufficient conditions on the order type of the base are given for the Boolean algebra to have the basis property.
\end{abstract}

Introduction. The notion of an independent family of sets was first introduced in [1] by Fichtenholz-Kantorovitch. Their results were generalized in [3] by Hausdorff where it was shown that, if $|I|=m$, there exists an independent family of subsets of $I$ of power $2^{m}$. It is well known that the free Boolean algebra on $\boldsymbol{m}$ generators is generated by an independent family of elements of power $\boldsymbol{m}$ and that every ultrafilter in this algebra has an independent set of generators. A weaker notion, that of an irredundant set of generators, or a weakly independent set of generators, has been considered by both A. Tarski [11] and I. Reznikoff [7] in the setting of mathematical logic, and it is the algebraic version of this notion which we call a basis for an ultrafilter. Boolean algebras in which every ultrafilter has a basis are said to have the basis property. The idea of an independent set modulo a filter has been used by K. Kunen in [4] and this leads to the property considered here, that of a basis over a filter.

The first section consists of the definitions and basic lemmas concerning the above mentioned three notions and a theorem showing that a large class of Boolean algebras do not have the basis property. In $\$ 2$ Boolean algebras with an ordered base are considered and for this class of Boolean algebras necessary and sufficient conditions on the order type of the base are given for the Boolean algebra to have the basis property. For these Boolean algebras, the latter two notions of a basis are shown to be equivalent and further, any such Boolean algebra with the basis property must have cardinality less than or equal to $2^{N_{0}}$. Finally a summary of the relationships between these three concepts of basis is given. 
Preliminaries. If $\mathfrak{A}$ is a Boolean algebra we assume $\mathfrak{A}=$ $\langle A, \vee, \wedge,-, 0,1\rangle$ and if $\mathscr{F}$ is a filter in $\mathfrak{A}$, and $a \in A$, we write $a / \mathscr{F}=$ $\bar{a}$. The basic results concerning Boolean algebras may be found in [2] or [10]. We recall that a filter in a Boolean algebra is generated by $\left\{b_{\nu}\right\}_{\nu<\alpha} \subset \mathscr{F}$ if for each $a \in \mathscr{F}$ there exists $\nu_{1}, \cdots, \nu_{k}<\alpha$ with $b_{\nu_{1}} \wedge \cdots \wedge b_{\nu_{k}} \leqq a$. A family of elements $\left\{a_{\nu}\right\}_{\nu<\alpha} \subset A$ is independent if, for all $\nu_{1}, \cdots, \nu_{n}<\alpha$ which are distinct, $b_{\nu_{1}} \wedge \cdots \wedge b_{\nu_{n}} \neq 0$ where for each $i$, $b_{\nu_{\mathrm{t}}}=a_{\nu_{\mathrm{i}}}$ or $-a_{\nu_{i}}$.

1. Definition 1.1. A filter $\mathscr{F}$ in a Boolean algebra $\mathfrak{A}$ is said to have a basis $\left\{a_{\nu}\right\}_{\nu<\alpha}$ if

(i) $\left\{a_{\nu}\right\}_{v<\alpha}$ generates $\mathscr{F}$, and

(ii) if $\nu_{0}, \cdots, \nu_{n+1}<\alpha$ are distinct, then $a_{\nu_{0}} \wedge \cdots \wedge a_{\nu_{n}} \not a_{\nu_{n+1}}$.

A Boolean algebra is said to have the basis property if each ultrafilter has a basis. The definition of basis is weaker than that of independent set of generators. For example, in the Boolean algebra of finite and cofinite subsets of $\omega$, there is only one nonprincipal ultrafilter and it has a basis but does not have an independent set of generators:

If $\left\{A_{n}\right\}_{n \in \omega} \subset \mathscr{F}$ is an independent set of generators then each $A_{n}$ is cofinite. Let $B_{0}=\left\{n_{1}, \cdots, n_{k}\right\}=\sim A_{0}$ and, for $i=1, \cdots, k$, let

$$
B_{i}=\left\{\begin{aligned}
A_{i} & \text { if } n_{i} \in A_{i} \\
\sim A_{i} & \text { otherwise }
\end{aligned}\right.
$$

Then $B_{0} \subset B_{1} \cup \cdots \cup B_{k}$. We may assume without loss of generality that $B_{0} \subset\left(\sim A_{1} \cup \cdots \cup \sim A_{j}\right) \cup\left(A_{j+1} \cup \cdots \cup A_{k}\right)$. Thus, since $B_{0}=$ $\sim A_{0}$, we have $\left(A_{1} \cap \cdots \cap A_{j}\right) \cap \sim A_{0} \subset\left(A_{j+1} \cup \cdots \cup A_{k}\right)$ and hence $A_{1} \cap \cdots \cap A_{j} \subset A_{0} \cup A_{j+1} \cup \cdots \cup A_{k}$ which contradicts the independence of the $A_{i}$ 's. On the other hand, the complements of singletons form a basis for $\mathscr{F}$.

Condition (ii) is an irredundancy condition and is the algebraic translation of the logical notion of an independent set of formulas, apparently first introduced by Tarski [11]. This algebraic version will be referred to as weak independence. In this connection, it is interesting to note that Reznikoff in [7] showed that every filter in a free Boolean algebra has a basis. The following notion of a basis for one filter over another filter is a modification of the definition of being independent modulo a filter (See [4]):

Definition 1.2. Let $\mathscr{G}$ and $\mathscr{F}$ be filters in the Boolean algebra $\mathfrak{A}$ with $\mathscr{F} \supsetneqq \mathscr{G} . \quad\left\{a_{\nu}\right\}_{v<\alpha} \subset \mathscr{F}$ is a basis for $\mathscr{F}$ over $\mathscr{G}$ if 
(i) $\mathscr{G} \cup\left\{a_{\nu}\right\}_{\nu<\alpha}$ generates $\mathscr{F}$ and

(ii) if $\nu_{0}, \cdots, \nu_{n+1}<\alpha$ are distinct, then $-a_{\nu_{0}} \vee \cdots \vee-$ $a_{\nu_{n}} \vee a_{\nu_{n+1}} \notin \mathscr{G}$.

In particular, condition (ii) allows one to extend $\mathscr{G}$ to a proper filter containing $a_{\nu_{0}} \wedge \cdots \wedge a_{\nu_{n}} \wedge-a_{\nu_{n+1}}$.

If $\mathscr{F}$ is a filter in a Boolean algebra $\mathfrak{A}$, and $\mathscr{I}=\{-x: x \in \mathscr{F}\}$ then $\mathscr{I}$ is an ideal and, by $\mathfrak{U} / \mathscr{F}$ we mean the quotient algebra $\mathfrak{U} / \mathscr{I}$.

LeMma 1.3. Let $\mathfrak{A}$ be a Boolean algebra, $\mathscr{F}$ an ultrafilter in $\mathfrak{A}, \mathscr{G}$ a filter in $\mathfrak{A}, \overline{\mathfrak{A}}=\mathfrak{U} / \mathscr{G}$ and $\overline{\mathscr{F}}=\{\bar{a}: a \in \mathscr{F}\}$. Then $\mathscr{F}$ has a basis over $\mathscr{F} \cap \mathscr{G}$ if and only if $\overline{\mathscr{F}}$ has a basis in $\overline{\mathfrak{A}}$.

Proof. It is straightforward to verify that $\left\{a_{\nu}\right\}_{\nu<\alpha}$ is a basis for $\mathscr{F}$ over $\mathscr{F} \cap \mathscr{G}$ if and only if $\left\{\bar{a}_{\nu}\right\}_{\nu<\alpha}$ is a basis for $\overline{\mathscr{F}}$ in $\overline{\mathfrak{A}}$.

Lemma 1.4. Let $\mathfrak{A}$ be an infinite Boolean algebra and $\left\{x_{n}\right\}_{n \in \omega}$ an infinite set of distinct ultrafilters in $\mathfrak{A}$. Then there exists an infinite subsequence $\left\{x_{n_{k}}\right\}_{k \in \omega} \subset\left\{x_{n}\right\}_{n \in \omega}$ and $\left\{a_{k}\right\}_{k \in \omega} \subset A$ of pairwise disjoint ele ments with $a_{k} \in x_{n k}$.

Proof. Let $b_{0} \in x_{0}$ with $-b_{0} \in x_{1}$ and let $B_{0}^{+}=\left\{x_{n}: b_{0} \in x_{n}\right\} B_{0}^{-}=$ $\left\{x_{n}:-b_{0} \in x_{n}\right\}$. If $\left|B_{0}^{+}\right|=\boldsymbol{\aleph}_{0}$, set $B_{0}=B_{0}^{+}, a_{0}=-b_{0}, c_{0}=b_{0}$ and $n_{0}=$ 1. If $\left|B_{0}^{+}\right| \neq \boldsymbol{N}_{0}$, set $B_{0}=B_{0}^{-}, a_{0}=b_{0}, c_{0}=-b_{0}$ and $n_{0}=0$. Suppose $\left\{a_{k}\right\}_{k \leqq m},\left\{c_{k}\right\}_{k \leqq m}\left\{x_{n k}\right\}_{k \leqq m}$ and $\left\{B_{k}\right\}_{k \leqq m}$ have been defined with $B_{k}$ infinite for $k \leqq m$ and $i<j \leqq m$ implies $B_{i} \supsetneqq B_{j}$ and for all $k \leqq m$ we have

(i) $a_{k} \in x_{n k}, c_{k} \in x$ for all $x \in B_{k}, a_{k} \wedge a_{i}=0$ if $k \neq i$ and $a_{k} \wedge c_{i}=0$ for all $k \leqq i$.

Let $x_{0}^{m}$ and $x_{1}^{m}$ be distinct elements of $B_{m}$ of the form $x_{n}$, where $n>n_{m}$, and let $b_{m} \in x_{0}^{m}$ with $-b_{m} \in x_{1}^{m}$. Then $c_{m} \wedge b_{m} \in x_{0}^{m}$ and $c_{m} \wedge-b_{m} \in$ $x_{1}^{m}$. Let $B_{m+1}^{+}=\left\{y \in B_{m}: c_{m} \wedge b_{m} \in y\right\}, B_{m+1}^{-}=\left\{y \in B_{n}: c_{m} \wedge-b_{m} \in y\right\}$. If $\left|B_{m+1}^{+}\right|=N_{0}$, let $B_{m+1}=B_{m+1}^{-}, a_{m+1}=c_{m} \wedge-b_{m}, c_{m+1}=c_{m} \wedge b_{m}$ and $x_{n_{m+1}}=$ $x_{1}^{m}$. If $\left|B_{m+1}^{+}\right| \neq \boldsymbol{N}_{0}$, let $B_{m+1}=B_{m+1}^{-}, a_{m+1}=c_{m} \wedge b_{m}, c_{m+1}=c_{m} \wedge-b_{m}$ and $x_{n_{m+1}}=x_{0}^{m}$. Clearly $\left\{x_{n_{k}}\right\}_{k \in \omega}$ and $\left\{a_{k}\right\}_{k \in \omega}$ so defined have the desired properties.

THEOREM 1.5. Let $\mathfrak{A}$ be a $\sigma$-complete Boolean algebra, $\mathscr{G}$ a filter in $\mathfrak{U}$ and $\mathscr{F}$ an ultrafilter in $\mathfrak{U}$ with $\mathscr{F} \supsetneqq \mathscr{G}$. Then $\mathscr{F}$ has a basis over $\mathscr{G}$ if and only if there exists $a b \in A$ such that $\mathscr{F}$ is generated by $\mathscr{G} \cup\{b\}$.

Proof. Obviously if $\mathscr{F}$ is generated by $\mathscr{G} \cup\{b\}$ then $\mathscr{F}$ has a basis over $\mathscr{G}$. Now suppose $\mathscr{F}$ has a basis $\left\{a_{\nu}\right\}_{\nu<\alpha}$ over $\mathscr{G}$ and that $\alpha$ is infinite (if $\alpha$ is finite, then clearly $\mathscr{G} \cup\{b\}$ generates $\mathscr{F}$ where $b=$ 
$\left.a_{0} \wedge a_{1} \wedge \cdots \wedge a_{\alpha-1}\right)$. Let $\mathscr{F}_{\nu}$ be an ultrafilter in $\mathfrak{A}$ such that $\mathscr{F}_{\nu} \supset \mathscr{G} \cup$ $\left\{a_{\mu}\right\}_{\mu<\alpha, \mu \neq \nu} \cup\left\{-a_{\nu}\right\}$. This is possible by the definition of a basis over $\mathscr{G}$.

(1) If $a \in \mathscr{F}$ then $\left\{\nu<\alpha: a \notin \mathscr{F}_{\nu}\right\}$ is finite. Since $a \in \mathscr{F}$ there exists $b \in \mathscr{G}$ and $\nu_{0}, \cdots, \nu_{n}<\alpha$ such that $b \wedge a_{\nu_{0}} \wedge \cdots \wedge a_{\nu_{n}} \leqq a$ and $a \in \mathscr{F}_{\nu}$ for all $\nu \neq \nu_{0}, \cdots, \nu_{n}$,

By Lemma 1.4 there exists a subsequence $\left\{\mathscr{F}_{\nu_{k}}\right\}$ of $\left\{\mathscr{F}_{\nu}\right\}_{\nu<\alpha}$ and $b_{k} \in \mathscr{F}_{v_{k}}$ such that $b_{k} \wedge b_{j}=0$ for $k \neq j$. Since $\mathfrak{A}$ is $\sigma$-complete there exist $b=\vee_{k \in \omega} b_{2 k}$ and $c=\vee_{k \in \omega} b_{2 k+1}$. Since $b \in \mathscr{F}_{\nu_{2 k}}$ for all $k$ we have $-b \notin \mathscr{F}$ by $(1)$ and therefore $b \in \mathscr{F}$. Similarly $c \in \mathscr{F}$. But $b \wedge c=0$ which contradicts $0 \notin \mathscr{F}$. Thus if $\mathscr{F}$ has a basis in $\mathfrak{A}$, the basis is finite.

COROLlary 1.6. Let $\mathfrak{A}$ be a $\sigma$-complete Boolean algebra and $\mathfrak{B}$ a homomorphic image of $\mathfrak{A}$. Then no nonprincipal ultrafilter in $\mathfrak{B}$ has a basis.

Proof. Immediate by the previous theorem and Lemma 1.3.

One easily sees that if $\mathfrak{A}$ has the basis property, this does not imply that, given an ultrafilter $\mathscr{F}$ extending a filter $\mathscr{G}, \mathscr{F}$ has a basis over $\mathscr{G}$. For example let $\mathfrak{A}_{m}$ be the free Boolean algebra on $\boldsymbol{m}$ generators and let $\mathfrak{B}$ be an atomless $\sigma$-complete Boolean algebra with $|\mathfrak{B}|<\boldsymbol{m}$. Then there exists a filter $\mathscr{G}$ in $\mathfrak{A}_{m}$ such that $\mathfrak{A}_{m} / \mathscr{G} \cong \mathfrak{B}$, but if $\mathscr{F}$ is an ultrafilter extending $\mathscr{G}$, then $\mathscr{F}$ has no basis over $\mathscr{G}$ by Lemma 1.3 and Corollary 1.6. It is interesting to note that for Boolean algebras with an ordered base, if an ultrafilter has a basis then it has a basis over every filter which it extends (see 2.5 and the remark preceding it). If an ultrafilter has a basis over every proper filter which it extends then it does have a basis since it has a basis over $\{1\}$. In fact if $\mathscr{G} \subset \mathscr{F}$, such that $\mathscr{F}$ has a basis over $\mathscr{G}$ and there exists $a \in \mathscr{F}$ with $a \leqq b$ for all $b \in \mathscr{G}$, then $\mathscr{F}$ has a basis in $\mathfrak{A}$.

COROLlaRy 1.7. Let $\mathfrak{A}$ be Boolean algebra and $\mathscr{F}$ an ultrafilter in $\mathfrak{A}$. If $\mathscr{F}$ has a basis over every proper subfilter, then $\mathscr{F}$ has a basis in $\mathfrak{A}$.

In addition to free Boolean algebras, it is well known that every countable Boolean algebra has the property that all nonprinciple ultrafilters have a basis.

The following lemma, probably first proved by Tarski [9] establishes this result:

LEMMA 1.8. Let $\mathscr{F}$ be an ultrafilter in a Boolean algebra $\mathfrak{A}$ such that $\mathscr{F}$ has a countable set $\left\{a_{n}\right\}_{n \in \omega}$ of generators. Then $\mathscr{F}$ has a basis. 
Proof. Assume without loss of generality that $a_{0} \neq 1$ and for $n<m$, $a_{n}>a_{m}$. Let $b_{n}=a_{n} \vee\left(\vee_{k<n}-a_{k}\right)$.

(1) $a_{n} \leqq b_{n}$ for all $n \in \omega$,

(2) $-a_{n} \leqq b_{m}$ for all $n<m$,

(3) $b_{n} \vee b_{m}=1$ for all $n \neq m$.

Now

(4) $\left\{b_{n}\right\}_{n \in \omega}$ is weakly independent if $\left\{b_{k_{1}} \wedge \cdots \wedge b_{k_{n}}\right\} \leqq b_{k_{n+1}}$ then $-\left(b_{k_{1}} \wedge \cdots \wedge b_{k_{n}}\right) \wedge b_{k_{n+1}}=1$.

But, since $b_{k_{\mathrm{r}}} \vee b_{k_{n+1}}=1$ for $1 \leqq i \leqq k$ by (3), we have $\left(b_{k_{1}} \wedge \cdots \wedge b_{k_{n}}\right) \vee b_{k_{n+1}}=1$. Since $b_{k_{n+1}} \neq 1$, this is a contradiction.

(5) $\left\{b_{n}\right\}_{n \in \omega}$ generates $\mathscr{F}$.

A simple inductive proof shows $a_{n}=\wedge_{i \leqq n} b_{i}$.

COROllary 1.9. Let $\mathfrak{A}$ be a $\sigma$-complete Boolean algebra and $\mathfrak{B}$ a homomorphic image of $\mathfrak{A}$. Then no nonprincipal ultrafilter in $\mathfrak{B}$ has a countable set of generators.

Proof. By 1.6 and 1.8 .

From 1.9, the well-known result that no infinite homomorphic image of a $\sigma$-complete Boolean algebra is countable is immediate.

The question of whether every projective Boolean algebra has the basis property is open. Since little is known about projective Boolean algebras a positive answer to this question would be most interesting. A characterization of those Boolean algebras with the basis property, or one for those ultrafilters with a basis - perhaps in terms of chains in the filter - are additional areas of investigation. These latter two problems are answered completely in the case of Boolean algebras with an ordered base in the next section.

2. In this section we restrict the discussion to Boolean algebras with an ordered base. These Boolean algebras were first introduced by Mostowski and Tarski in [6] and have been studied more recently by Mayer and Pierce [5] and Rotman [8] where additional references may be found. Rotman shows that in a Boolean algebra with an ordered base there are at most countably many independent elements. The question for weakly independent elements appears to be open.

Definition 2.1. A Boolean algebra $\mathfrak{A}$ has an ordered base $X$ if $X$ is linearly ordered by $<$ (the order in $\mathfrak{A}$ ), $X$ generates $\mathfrak{A}, 0 \in X$ and $1 \notin X$.

If $(A, \leqq)$ is a linearly ordered set, then the cofinality of $A(\operatorname{cf}(A))$ is 
$\inf \{|B|$ : for all $a \in A$ there exists $b \in B \subset A$ with $a \leqq b\}$. The coinitiality of $A(\operatorname{ci}(A))$ is the $\inf \{|B|:$ for all $a \in A$ there exists a $b \in B \subset A$ with $b \leqq a\}$. An initial segment of $A$ is a set $B \subset A$ such that if $b \in B$ and $a<b$ then $a \in B$. A tail of $A$ is the complement of an initial segment.

LEMMA 2.2. Let $\mathfrak{A}$ be a Boolean algebra with ordered base $X, Y$ an initial segment of $X$ and $\mathscr{F}$ an ultrafilter in $\mathfrak{A}$ containing $\{-y ; y \in Y\} \cup$ $(X \sim Y)$. If $x \in \mathscr{F}$, then there exists $y \in Y$ and $z \in X \sim Y$ such that $x \geqq-y \wedge z$.

Proof. Since $X$ is a set of generators for the Boolean algebra and $\mathscr{F}$ is an ultrafilter, the conclusion is obvious.

THEOREM 2.3. If $\mathfrak{A}$ is a Boolean algebra with ordered base $X$, and there exists an initial segment $Y \subset X$ with $\operatorname{cf}(Y)>N_{0}$ or there exists a tail $Z \subset X$ with $\operatorname{ci}(Z)>\aleph_{0}$, then $\mathfrak{U}$ does not have the basis property of ultrafilter.

Proof. We may assume there exists an initial segment $Y \subset X$ with $\operatorname{cf}(Y)>\boldsymbol{N}_{0}$, for otherwise $\{0\} \cup\{-x: x \in X \sim\{0\}\}$ is an ordered basis with an initial segment $Z$ having cofinality greater than $\boldsymbol{N}_{0}$. Let $Y=\left\{a_{i}\right\}_{i \in I}$ and let $\mathscr{F}$ be an ultrafilter such that $\mathscr{F} \supset\left\{-a_{l}\right\}_{i \in I}$ and $\mathscr{F} \supset X-Y$. Suppose $\mathscr{F}$ has a basis $\left\{c_{\nu}\right\}_{\nu<\lambda}$.

(1) $|\lambda|>\boldsymbol{N}_{0}$ - we first note that no finite meet $d$ of basis elements is less than or equal to all $-a_{t}$, for otherwise by Lemma 2.2 there exists $-a_{\jmath}$ and $x \in X-Y$ with $-a_{1} \wedge x \leqq d \leqq-a_{i}$ for all $i \in I$. Hence $-a_{l} \wedge x \leqq-a_{\imath} \wedge x$ for all $i \in I$. Choosing $a_{i}>a_{j}$ we have $a_{i}<x$ since $Y$ is an initial segment of $X$. Thus $a_{i} \wedge-a_{j} \wedge x=a_{i} \wedge-a_{j}=0$ so $a_{i} \leqq a_{j}$, a contradiction.

By the above argument, $|\lambda| \geqq \boldsymbol{\aleph}_{0}$, so assume $|\lambda|=\boldsymbol{\aleph}_{0}$. Let $d_{n}=$ $c_{0} \wedge \cdots \wedge c_{n}$. Again by above argument, for each $n \in \omega$ there exists $-a_{i_{n}}$ such that $d_{n}-a_{i_{n}}$. Since $\operatorname{cf}(Y)>\boldsymbol{N}_{0}$, we arrive at an obvious contradiction - hence (1) is established.

(2) $\mathscr{F}$ has no basis.

Case 1. $\operatorname{ci}(X \sim Y)>\boldsymbol{N}_{0}$.

Let $\left\{b_{j}\right\}_{j \in J}=X \sim Y$. By Lemma 2.2 there exists $i_{0} \in I$ and $j_{0} \in J$ with $c_{0} \geqq-a_{i_{0}} \wedge b_{j_{0}} \geqq d_{0}$ where $d_{0}$ is a finite meet of the basis elements $\left\{c_{\nu}\right\}_{\nu<\lambda}$. By Lemma 2.2, choose $i_{1} \in I$ and $j_{1} \in J$ such that $-a_{i_{1}} \wedge b_{j_{1}}<d_{0}$. Proceeding in this manner we construct $c_{0} \geqq-a_{i_{0}} \wedge b_{i_{0}} \geqq d_{0} \geqq \cdots \geqq$ 
$-a_{i_{n}} \wedge b_{i_{n}} \geqq d_{n} \geqq \cdots$ where $-a_{i_{n}} \wedge b_{i_{n}}>-a_{i_{n+1}} \wedge b_{i_{n+1}}$ and $d_{n}$ is a finite meet of basis elements. Since $\operatorname{cf}(Y)>\boldsymbol{N}_{0}$, there exists $a \in Y$ with $-a \leqq-a_{i_{n}}$ for all $n \in \omega$ and $b \in X \sim Y$ with $b \leqq b_{i_{n}}$ for all $n \in \omega$. Hence $-a \cdot b \leqq d_{n}$ for all $n \in \omega$. Now there exist $\nu_{1}, \cdots, \nu_{k}$ with $c_{\nu_{1}} \wedge \cdots \wedge c_{\nu_{k}} \leqq-a \cdot b \leqq d_{n}$ for all $n \in \omega$. Since the $d_{n}$ 's are strictly decreasing, there exists $c_{\nu_{0}}$ occurring in some $d_{n_{0}}$ with $c_{\nu 0} \neq c_{\nu_{1}}, \cdots, c_{v_{k}}$. Hence $c_{\nu_{1}} \wedge \cdots \wedge c_{\nu_{k}} \leqq d_{n_{0}}<c_{\nu 0}$ contradicting the weak independence of the $c_{\nu}$ 's.

Case 2. $\operatorname{ci}(X \sim Y) \leqq \boldsymbol{N}_{0}$.

We observe that for each $a \in Y$ and $b \in X \sim Y, \mid\left\{c_{v}:-a \wedge b \leqq\right.$ $\left.c_{\nu}\right\} \mid<\boldsymbol{N}_{0}$ - for otherwise there exist $c_{\nu_{1}}, \cdots, c_{\nu_{k}}$ with $c_{\nu_{1}} \wedge \cdots \wedge c_{\nu_{k}} \leqq$ $-a \wedge b$ with $-a \wedge b$ less than infinitely many $c_{\nu}$ 's which contradicts the weak independence of the $c_{v}$ 's. Let $\left\{b_{n}\right\}_{n \in \omega}$ be coinitial with $X \sim$ $Y$. Let $B_{n}=\left\{c_{v}:-a_{\imath} \wedge b_{n} \leqq c_{v}\right.$ for some $\left.i \in I\right\}$. Since $\bigcup_{n \in \omega} B_{n}=$ $\left\{c_{\nu}\right\}_{\nu<\lambda}$ and $\lambda$ is uncountable there is an $n_{0}$ with $B_{n_{0}}$ infinite. This implies there is a countable $I_{0} \subset I$ such that $\mid\left\{c_{\nu}:-a_{\imath} \wedge b_{n_{0}} \leqq c_{\nu}\right.$ for some $\left.i \in I_{0}\right\} \mid \geqq$ $\boldsymbol{\aleph}_{0}$. Since $\operatorname{cf}(Y)>\boldsymbol{N}_{0}$ there exists $-a \in Y$ with $-a \leqq-a_{i}$ for all $i \in I_{0}$. Hence $-a \wedge b_{n_{0}}$ is less than or equal to infinitely many basis elements - a contradiction.

THEOREM 2.4. Let $\mathfrak{A}$ be a Boolean algebra with ordered base $X$. If $\operatorname{cf}(Y) \leqq \boldsymbol{N}_{0}$ for every initial segment $Y$ of $X$ and $c_{i}(Z) \leqq \boldsymbol{N}_{0}$ for every tail $Z$ of $X$, then $\mathfrak{A}$ has the basis property for ultrafilters.

Proof. Let $\mathscr{F}$ be an ultrafilter in $\mathfrak{A}$. Let $Y=\{y \in X: y \notin \mathscr{F}\}$. Then $Y$ is an initial segment of $X$ and, by Lemma $2.2, \mathscr{F}$ is generated by $\{-y: y \in Y\} \vee(X \sim Y)$. By hypothesis there is a countable sequence $\left\{x_{n}\right\}$ which is coinitial in $X \sim Y$ and a countable sequence $\left\{y_{n}\right\}$ which is cofinal in $Y$. Clearly $\mathscr{F}$ is generated by $\left\{-y_{n}\right\} \cup\left\{x_{n}\right\}$. Therefore, by Lemma 1.8 $\mathscr{F}$ has a basis.

Theorems 2.3 and 2.4 completely characterize the order types of ordered bases which give rise to Boolean algebras with the basis property for ultrafilters.

Boolean algebras with an ordered base which have the basis property for ultrafilters have in fact a stronger property - namely, every ultrafilter has a basis over every filter which it contains (see remark following 1.6). This is established by Lemma 1.3 and the following:

COROllary 2.5. Let $\mathfrak{A}$ be a Boolean algebra with ordered base $X$ and suppose $\mathfrak{A}$ has the basis property for ultrafilters. If $\mathfrak{B}$ is a homomorphic image of $\mathfrak{U}$ then $\mathfrak{B}$ has the basis property for ultrafilters. 
Proof. Let $h: \mathfrak{A} \rightarrow \mathfrak{B}$ be a homomorphism and let $X^{\prime}=h(X) \sim\{1\}$. Then one easily checks that $X^{\prime}$ is an ordered base for $\mathfrak{B}$. By 2.4 it suffices to check that if $Y^{\prime}$ is any initial segment of $X^{\prime}$ and $Z^{\prime}$ is any tail of $X^{\prime}$, then $\operatorname{cf}\left(Y^{\prime}\right) \leqq \boldsymbol{N}_{0}$ and $\operatorname{ci}\left(Z^{\prime}\right) \leqq \boldsymbol{N}_{0}$. Since $Y=\left\{y \in X: h(y) \in Y^{\prime}\right\}$ has cofinality $\leqq \boldsymbol{N}_{0}$ by 2.3 , it is easy to verify that $\operatorname{cf}\left(Y^{\prime}\right) \leqq \boldsymbol{N}_{0}$. Similarly, one sees that $\operatorname{ci}\left(Z^{\prime}\right) \leqq \boldsymbol{N}_{0}$.

By the proof of 2.3 and 2.4 it is clear that if $\mathfrak{A}$ is a Boolean algebra with an ordered basis $X$ and $\mathscr{F}$ is an ultrafilter in $\mathscr{A}$ then $\mathscr{F}$ has a basis iff $\operatorname{cf}(Y) \leqq \boldsymbol{N}_{0}$ and $\operatorname{ci}(Z) \leqq \boldsymbol{N}_{0}$ where $Y=\{x \in \mathscr{F}:-x \in X\}$ and $Z=$ $\{x \in \mathscr{F}: x \in X\}$. Similarly, as in 2.5 , if $\mathscr{F}$ has a basis and $\mathscr{G} \subset \mathscr{F}$, then $\overline{\mathscr{F}}$ has a basis in $\mathfrak{A} / \mathscr{G}$. Combined with 1.8 this gives us

COROllary 2.6. If $\mathfrak{A}$ is a Boolean algebra with an ordered base and $\mathscr{F}$ is an ultrafilter in $\mathfrak{U}$ then the following are equivalent:

(i) $\mathscr{F}$ has a basis

(ii) $\mathscr{F}$ has a basis over every filter $\mathscr{G} \subset \mathscr{F}$.

THEOREM 2.7. Let $\mathfrak{A}$ be a Boolean algebra with an ordered base $X$. If $\mathfrak{U}$ has the basis property for ultrafilters, then $|\mathfrak{U}| \leqq 2^{\aleph_{0}}$.

Proof. Let $X^{*}$ be the completion by cuts of $X$. Then $X^{*}$ is a compact, first countable Hausdorff space under the order topology and hence has cardinality $\leqq 2^{\aleph_{0}}$ (see [9]). As a consequence $\mid \mathfrak{A} \leqq 2^{\boldsymbol{\aleph}_{0}}$.

We summarize results concerning our three notions of basis in the following table where we use the notation:

(I) $\mathscr{F}$ has an independent set of generators

(II) $\mathscr{F}$ has a basis

(III) $\mathscr{F}$ has a basis over every proper subfilter.

\begin{tabular}{|c|c|c|}
\hline & $\begin{array}{l}\text { Boolean } \\
\text { algebras }\end{array}$ & $\begin{array}{c}\text { Boolean algebras with } \\
\text { an ordered base }\end{array}$ \\
\hline $\mathrm{I} \rightarrow \mathrm{II}$ & Yes & Yes \\
\hline $\mathrm{II} \rightarrow \mathrm{III}$ & No & Yes \\
\hline $\mathrm{III} \rightarrow \mathrm{I}$ & No & No \\
\hline $\mathrm{I} \rightarrow \mathrm{III}$ & No & Yes \\
\hline $\mathrm{II} \rightarrow \mathrm{I}$ & No & No \\
\hline $\mathrm{III} \rightarrow \mathrm{II}$ & Yes & Yes \\
\hline
\end{tabular}




\section{REFERENCES}

1. G. Fichtenholz, and L. Kantorovitch, Sur les opérations linéairs dans l'espace des fonctions bornées, Studia Math., 5 (1934), 69-98.

2. P. Halmos, Lectures on Boolean Algebras, van Nostrand Mathematical Studies No. 1, Princeton, 1963.

3. F. Hausdorff, Über zwei Satze von G. Fichtenholz und L. Kantorovitch, Studia Math., 6 (1936), $18-19$.

4. K. Kunen, Ultrafilters and independent sets, Trans. Amer. Math. Soc., 172 (1972), 299-306.

5. R. D. Mayer and R. S. Pierce, Boolean algebras with ordered bases, Pacific J. Math., 10 (1960), 925-942.

6. A. Mostowski and A. Tarski, Boolesche Ringe mit geordneter Basis, Fund. Math., 32 (1939), 69-86.

7. I. Reznikoff, Tout ensemble de formules de la logique classique est équivalent à un ensemble indépendant, C. R. Acad. Sc., Paris (1) 260 (1965), 2385-2388.

8. B. Rotman, Boolean algebras with ordered bases, Fund. Math., 75 (1972), 186-197.

9. Prabir Roy, The cardinality of first countable spaces, Bull. Amer. Math. Soc., 77 (1971), 1057-1059.

10. R. Sikorski, Boolean Algebras, Springer Verlag, New York, 1969.

11. A. Tarski, Über einige fundamentale Begriffe der Metamathematik, C. R. Soc. Sc. Lettres Varsouie, classe III, 23 (1930), 22-29.

Received June 26, 1975, and in revised form November 3, 1975.

EMORY UNIVERSITY 




\section{Pacific Journal of Mathematics}

\section{Vol. 62, No. $1 \quad$ January, 1976}

Mieczyslaw Altman, Contractor directions, directional contractors and

directional contractions for solving equations . .................. 1

Michael Peter Anderson, Subgroups of finite index in profinite groups .........

Zvi Arad, Abelian and nilpotent subgroups of maximal order of groups of odd order

John David Baildon and Ruth Silverman, On starshaped sets and Helly-type theorems ..........................................

John W. Baker and R. C. Lacher, Some mappings which do not admit an

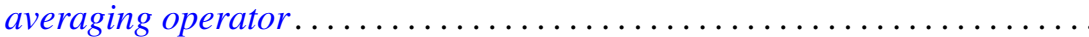

Joseph Barback, Composite numbers and prime regressive isols . . . . . . . . . .

David M. Boyd, Composition operators on $H^{p}(A) \ldots \ldots \ldots \ldots \ldots \ldots \ldots$

Maurice Chacron, Co-radical extension of PI rings . . . . . . . . . . . . .

Fred D. Crary, Some new engulfing theorems . . . . . . . . . . . . . . .

Victor Dannon and Dany Leviatan, A representation theorem for convolution transform with determining function in $L^{p} \ldots \ldots \ldots \ldots \ldots \ldots \ldots \ldots \ldots \ldots \ldots \ldots \ldots \ldots$

Mahlon M. Day, Lumpy subsets in left-amenable locally compact

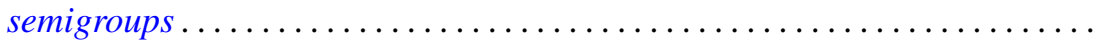

Michael A. Gauger, Some remarks on the center of the universal enveloping algebra of a classical simple Lie algebra . .

David K. Haley, Equational compactness and compact topologies in rings

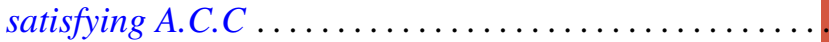

Raymond Heitmann, Generating ideals in Prüfer domains .

Gerald Norman Hile, Entire solutions of linear elliptic equations with

Laplacian principal part. .

Richard Oscar Hill, Moore-Postnikov towers for fibrations in which $\pi_{1}$ (fiber) is non-abelian

John Rast Hubbard, Approximation of compact homogeneous maps . .

Russell L. Merris, Relations among generalized matrix functions . .

V. S. Ramamurthi and Edgar Andrews Rutter, On cotorsion radicals ...

Ralph Tyrrell Rockafellar and Roger Jean-Baptiste Robert Wets, Stochastic

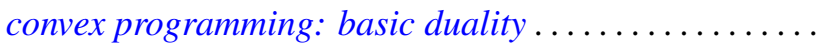

Alban J. Roques, Local evolution systems in general Banach spaces ..

I. Bert Russak, An indirect sufficiency proof for problems with bounded state variables.

Richard Alexander Sanerib, Jr., Ultrafilters and the basis property. .

H. A. Seid, The decomposition of multiplication operators on $L_{p}$-spaces . .

Franklin D. Tall, The density topology .................. 